\title{
Traceable calibration of a horizontally polarised reference antenna with omnidirectional pattern at VHF frequencies for ILS field strength validation
}

\author{
T. Schrader ${ }^{1}$, T. Kleine-Ostmann ${ }^{1}$, and J. Bredemeyer ${ }^{2}$ \\ ${ }^{1}$ Physikalisch-Technische Bundesanstalt (PTB), Bundesallee 100, 38116 Braunschweig, Germany \\ ${ }^{2}$ Flight Calibration Services FCS GmbH, Hermann-Blenk-Straße 32 A, 38108 Braunschweig, Germany \\ Correspondence to: T. Schrader (thorsten.schrader@ptb.de)
}

\begin{abstract}
We present a traceable calibration of a specially designed horizontally polarised reference antenna with an omnidirectional pattern in the E-plane for the frequency range between $105 \mathrm{MHz}$ and $120 \mathrm{MHz}$. This antenna is used as a validation tool for absolute field strength measurements at the localizer transmitter of an instrument landing system (ILS) at airports and is carried by a helicopter. We investigate whether we can treat it as a dipole-like antenna in the calibration setup despite its disk-shape body. We also investigate the suitability of an anechoic chamber for antenna calibration though it was not designed for that purpose. The measurements are based on scattering parameters (S-parameters) which we apply in the 3-antenna-method (TAM or 3-AM) to obtain the antenna gain and the antenna factor, respectively. An uncertainty budget for the antenna gain calibration is derived. We also report on the first practical application of the calibrated reference antenna.
\end{abstract}

\section{Introduction}

Terrestrial instrument landing systems (ILS) supporting air traffic management and navigation close to airports are subject to regular flight inspection (FI), where, in addition to other values of interest, absolute electric field strength values have to be determined according to the International Civil Aviation Organisation (ICAO 2000 and 2006). For this purpose the flight inspection service providers utilize medium size FI aircrafts equipped with navigation receivers and omnidirectional antennas mounted on the upper fuselage of the aircraft.
In order to validate the measured field strength values obtained during flight inspection, we developed a new method being totally independent of the aircrafts typically used. In our method, we use a helicopter carrying an autonomous payload on its external load hook, which consists of the new reference antenna and the receiving/recording system (Bredemeyer et al., 2012). The latter contains a Rohde \& Schwarz EVS 300 navigation receiver, a global positioning system (GPS) receiver, a data storage system, and a battery unit. Nylon ropes with $8 \mathrm{~m}$ length provide the required clearance between the lower fuselage of the helicopter and the reference antenna and between the antenna and the receiving/recording system, respectively. As the whole equipment is likely to swing underneath the helicopter during the flight and, moreover, may also rotate around the load hook, a special antenna was designed which provides an omnidirectional pattern in the E-plane. The antenna also shows a null in its sensitivity diagram along the vertical axis. Thus, it reduces the fringing effects on the electromagnetic field caused by the helicopter, the coaxial cable, and by the instrumentation box, respectively. The desired frequency range is $105 \mathrm{MHz}$ to $120 \mathrm{MHz}$, the co-polar receive mode of the antenna matches the horizontally polarized ILS LOC signal (localizer, indicates the lateral displacement of the aircraft to the landing runway).

The outer shape of the antenna is a flat disk with a diameter of approximately $70 \mathrm{~cm}$ and a height of approximately $3 \mathrm{~cm}$. The signal picked up by the antenna is fed into a coaxial cable which is routed along the vertical axis down to the receiver box. Thus, the antenna and its diagram are symmetrically with respect to the vertical axis and the coaxial cable does not interfere much with the antenna diagram. The $R \& S$ EVS 300 navigation receiver features an additional intermediate frequency (IF) output which provides a full channel 
band-pass signal. This is sampled at a high data rate and is directly recorded without any preprocessing. Thereby, the raw band-pass signal-in-space covers the complete channel bandwidth and allows for a maximum opportunity for any signal post-processing in order to extract the essential parameters of interest. The sampling electronics was developed by the authors and is based on field programmable gate arrays (FPGAs).

To calculate the electrical field strength from the antenna factor and the input signal of the receiver, the whole instrumentation has to be calibrated. The measurements to be taken later are performed in the far-field of the LOC, so we need to provide the far-field gain of the antenna and the antenna pattern diagrams in the E- and the H-plane. Hence, we discuss how to obtain the antenna gain from which we deduce the antenna factor traceable to the SI units. We also expand on the measurement uncertainty budget as ICAO (2000 and 2006) limits the total measurement uncertainty. The requirements by ICAO (2000 and 2006) state an overall uncertainty of the field strength measurements to be smaller than $3 \mathrm{~dB}$ for an absolute value of the electric field strength of $40 \mu \mathrm{V} \mathrm{m}^{-1}$. Taking these requirements into account we assume that the uncertainty contribution of the antenna factor should be in the order of $1 \mathrm{~dB}$ or less.

\section{Measurements}

Absolute antenna gain calibrations typically employ the 2 antenna (2-AM) or 3-antenna method (3-AM) in free-space and under far-field conditions. The 3-AM may be often found as the TAM in literature. But, in order to introduce a nonambiguous abbreviation for the 2-AM and 3-AM, we do not use TAM here. Two single or three pairs of similar sized antennas are placed in an echo-free environment and the transmission parameters are measured as a frequency response for each pair. Using Friis' formula (Balanis, 1982) for antennas separated by a distance $R$ with

$$
R>2 D^{2} f / c,
$$

where $D$ is the largest dimension of either antenna. Here $D=1.4 \mathrm{~m}, f=110 \mathrm{MHz}$, and $R$ should be larger than 1.45 , which is fulfilled for $R=3 \mathrm{~m}$ ), $c$ is the speed of light, and $f$ the frequency. Assuming a polarisation match and maximum reception alignment, the gain $G_{i}(f)$ in $\mathrm{dB}$ of each of the three antennas can be calculated according to Eq. (2ac). Here, we only have dipole-like or biconical antennas, where their phase center is located in the center axis of the antenna and does not vary with frequency. Using the freespace pathloss $\operatorname{PL}(f)=c^{2} /(4 \pi R f)^{2}$ and let $a_{j} a_{k}(f)$ be the measured linear S-parameter (forward transmission) between antenna $j$ and antenna $k$, the gain $G_{i}(f)$ is calculated using Eq. $(2 a-c)$.

$$
\begin{aligned}
& G_{1}(f)=10 \mathrm{~dB} \cdot \log _{10}\left(\sqrt{\frac{a_{1} a_{2}(f) \cdot a_{1} a_{3}(f)}{a_{2} a_{3}(f) \cdot \operatorname{PL}(f)}}\right) \text { in } \mathrm{dB} \\
& G_{2}(f)=10 \mathrm{~dB} \cdot \log _{10}\left(\sqrt{\frac{a_{2} a_{3}(f) \cdot a_{1} a_{2}(f)}{a_{1} a_{3}(f) \cdot \operatorname{PL}(f)}}\right) \text { in } \mathrm{dB} \\
& G_{3}(f)=10 \mathrm{~dB} \cdot \log _{10}\left(\sqrt{\frac{a_{1} a_{3}(f) \cdot a_{2} a_{3}(f)}{a_{1} a_{2}(f) \cdot \operatorname{PL}(f)}}\right) \text { in } \mathrm{dB}
\end{aligned}
$$

When using only two antennas, they have to be identical. In this case, the gain $G_{\mathrm{TX}, \mathrm{RX}}$ can be calculated using Eq. (3). The indices TX and RX indicate the transmit and receive mode of the antennas, respectively.

$$
G_{\mathrm{TX}, \mathrm{RX}}(f)=\frac{1}{2}\left[20 \cdot \log _{10}\left(a_{1} a_{2}(f)\right)-10 \cdot \log _{10} \mathrm{PL}(f)\right]
$$

From the results of Eq. (2a-c) we calculate the antenna factor $\mathrm{AF}_{i}(f)$ in $\mathrm{dB} \mathrm{m}^{-1}$ according to Eq. (4), where $G_{i}(f)$ is the gain in $\mathrm{dB}, Z_{0}=377 \Omega$ is the free-space wave impedance, and $Z=50 \Omega$ is the characteristic line impedance. The antenna factor AF is defined as the ratio of the incident electromagnetic field strength to the voltage $V$ on the line connection of an antenna with a specified impedance. For an electric field antenna the antenna factor AF has the unit $1 / \mathrm{m}$.

$$
\mathrm{AF}_{i}=20 \mathrm{~dB} \mathrm{~m}^{-1} \cdot \log _{10}\left(\frac{2 f}{c} \cdot \sqrt{\frac{\pi Z_{0}}{\left.Z \cdot 10^{G_{i}(f)}\right|_{\text {in dB }} / 10}}\right)
$$

$\mathrm{dB} \mathrm{m}^{-1}$

Employing S-parameters for the transmission measurements ensures that the antenna's input impedance is taken into account. Proximity effects and multipath interference, mutual coupling and multiple reflections must also be taken into account, when the measurement uncertainty budget is set up.

In order to take into account these parameters influencing the measurement uncertainty, we used the setup in our large anechoic chamber described in the following. Instead of using the center line of the chamber we slightly tilted the boresight axis between the antennas. In addition we positioned the setup in such a way that the distance to all possibly reflective installations including the absorber-lined walls, ground and ceiling of the anechoic chamber is maximized. The distance between the antennas and the supporting masts was at least $1 \mathrm{~m}$. We guided the coaxial cables loaded with ferrites at least $2 \mathrm{~m}$ behind the antenna and then routed them down to the ground. Alignment and polarisation of the antennas were carefully checked using vertical and horizontal laser lines. The distance was also measured applying laser lines which indicate the antenna positions on the ground, where we then used a calibrated measuring tape. The minimum distance between the antennas was chosen to $3 \mathrm{~m}$. 
After performing a system-error correction on the vector network analyser (VNA), we measured attenuators for which we hold calibration certificates covering the whole dynamic range of interest (e.g. -10 to $-50 \mathrm{~dB}$ ). Comparing the actual measurement results of the attenuation (here scattering parameter $\left.S_{21, \text { act }}\right)$ with those of the certificates $\left(S_{21, \text { cal }}\right)$ employing the $\mathrm{E}_{n}$-criteria (Wöger, 1999) in Eq. (5) we found a good agreement within the specified expanded uncertainties $U$ (expansion factor $k=2$ ). $U_{\text {cal }}$ is taken from the calibration certificate, $U_{\text {act }}$ is the measurement uncertainty specified for the actual experiment. Comparing attenuation and mismatch measurements after repeating the setup a week later we found $0.02 \mathrm{~dB}$ and $0.03 \mathrm{~dB}$ deviations, respectively.

$E_{n} \leq\left|\frac{\left|S_{21, \mathrm{act}}\right|-\left|S_{21, \mathrm{cal}}\right|}{\sqrt{U_{S 21, \mathrm{act}}^{2}+U_{S 21, \mathrm{cal}}^{2}}}\right|$

Using several mismatches instead of attenuators, also the measured input reflection coefficients were compared to the results from calibration certificates. From these validations we regard the S-parameters measured with the VNA as traceable to the SI units. The uncertainty of S-parameter measurements is within the specified and validated range. After all measurements we finally repeated the validation measurements to ensure the proper functionality of our VNA throughout all experiments described here.

\section{Validation}

Antenna calibrations in the VHF frequency range are typically carried out for dipole-like structures, e.g. biconical or logarithmic-periodic antennas. The antenna under test (AUT) here is more like a magnetic type of antenna, so we had to investigate (A) whether we could treat this AUT like a dipole or not. The second issue (B) to be solved during this project was the applicability of our anechoic chamber in antenna calibration in the VHF frequency range, though the anechoic chamber was originally not designed for that purpose. In near-field testing a combination of free-space loss and absorber reflectivity should add up to $-60 \mathrm{~dB}$ (Newell, 1988). According to Hemming (2002) absorbers should provide $-30 \mathrm{~dB}$ to $-40 \mathrm{~dB}$ reflectivity level or even better to ensure that the chamber has a negligible effect on the antenna measurements. When we performed the measurements described here, the CISPR 161-6 was not published and the guidance about antenna calibration test sites (CALTS) in CISPR 16-1-5 was not applicable. Therefore, we had to come up with our own procedure to validate the results taking into account case (A) and (B). We do not apply the very stringent requirements here which are needed for near-field measurements. We further know from other experiments, that the absorbers do not fulfill fairly high requirements for antenna calibration facilities. But, in some cases antenna measurements are still consistent with freespace measurements, which we verified experimentally. As

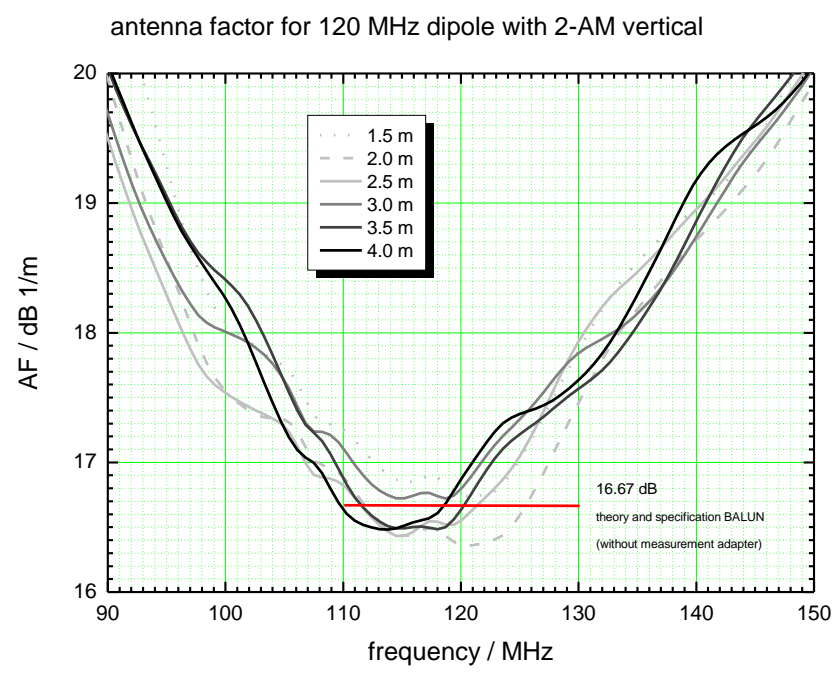

Fig. 1. AF for a set of reference dipoles as a function of distance.

a general requirement we keep the size of the antennas in the same order of medium mechanical dimensions (e.g. length $<1.4 \mathrm{~m})$.

\subsection{Validation of the anechoic chamber}

In order to deal with case (B) we applied the 2-AM and the 3AM. For validation of our procedures and the measurement results we chose two identical reference dipoles and two sets of biconical antennas for which we hold several calibration certificates from accredited antenna calibration laboratories. Those employed different methods to obtain the free-space antenna gain and antenna factor, e.g. the 3-AM method (3 m distance applying reference dipoles and biconical antennas) and the standard site method (SSM) using both an open area test site (OATS) and a semi-anechoic chamber (SAC) with $10 \mathrm{~m}$ distance. The specifications from the manufacturer obtained for $3 \mathrm{~m}$ distance are given as well. Thus, we compared antenna gains determined by measurements (for several measuring distances between $1.5 \mathrm{~m}$ and $4 \mathrm{~m}$ ) in our anechoic chamber with theoretical values of the antenna gain of the reference dipoles and, furthermore, we compared the antenna gain obtained from measurements in our chamber with a set of antenna gains obtained by accredited antenna calibration laboratories for the same identical antenna. Even more, we applied the 2-antenna-method and the 3-antenna-method (including one antenna with calibration certificate) to the reference dipoles and made a consistency check. The deviations found are within the specified uncertainty.

Figure 1 shows the AF as a function of frequency for a set of reference dipoles. We applied the 2-AM with the distance between the antennas as the parameter. Varying the distance from $1.5 \mathrm{~m}$ to $4 \mathrm{~m}$, the AF changes a few tenth of a $\mathrm{dB}$. Figure 2 shows a comparison of the AF for a reference dipole obtained from the 2-AM and from the 3-AM. The 
antenna factor $120 \mathrm{MHz}$ dipole 3-AM and 2-AM vertical $\mathrm{h}=3.5 \mathrm{~m}$

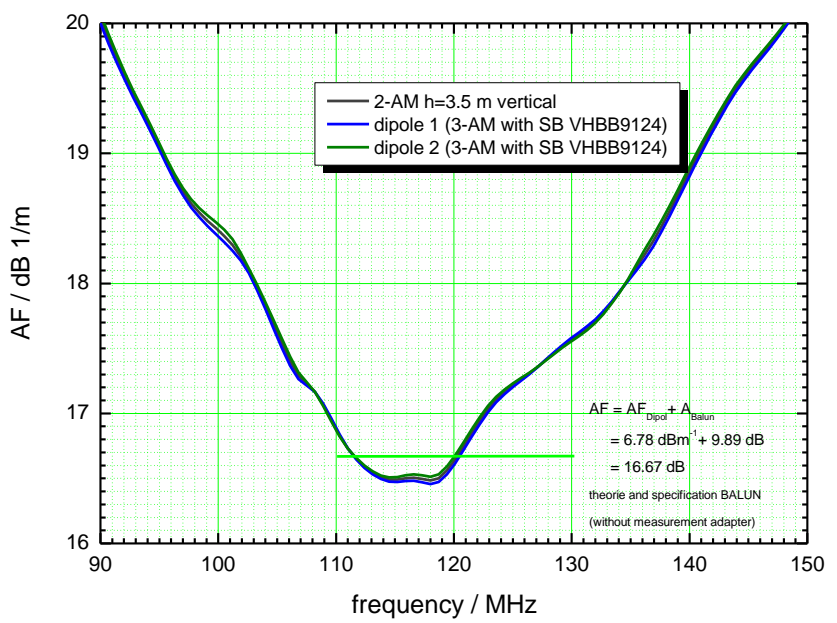

Fig. 2. Deviation of antenna gain of reference dipole obtained from 2-antenna-method and 3-antenna-method. The theoretical value of the $\mathrm{AF}$ is applicable at $120 \mathrm{MHz}$.

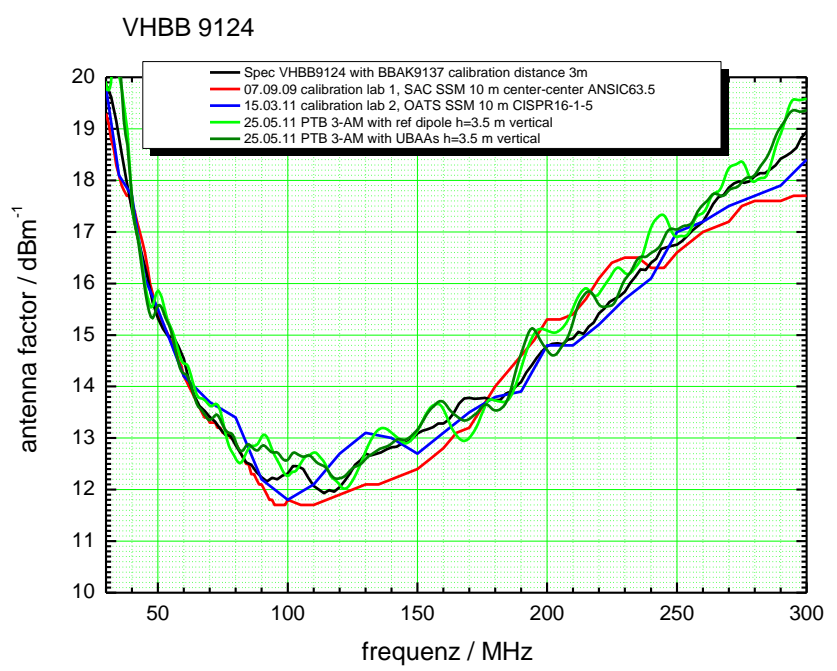

Fig. 3. Comparison of antenna factors from different accredited calibration laboratories and of PTB.

deviation found is about $0.1 \mathrm{~dB}$. Figure 3 shows a comparison of the free-space antenna factor for an antenna VHBB 9124 balun with BBAK 9137 biconical elements (manufacturer Schwarzbeck, Germany) provided from several calibration laboratories and by PTB (measurements obtained in anechoic chamber) employing different methods of antenna calibration. The deviation found for $120 \mathrm{MHz}$ is approximately $1 \mathrm{~dB}$.

As a result of the procedure applied we validated our antenna measurement setup in the frequency range of $105 \mathrm{MHz}$ to $120 \mathrm{MHz}$. The antenna gain values obtained are consistent to those from accredited laboratories within the specified uncertainty.

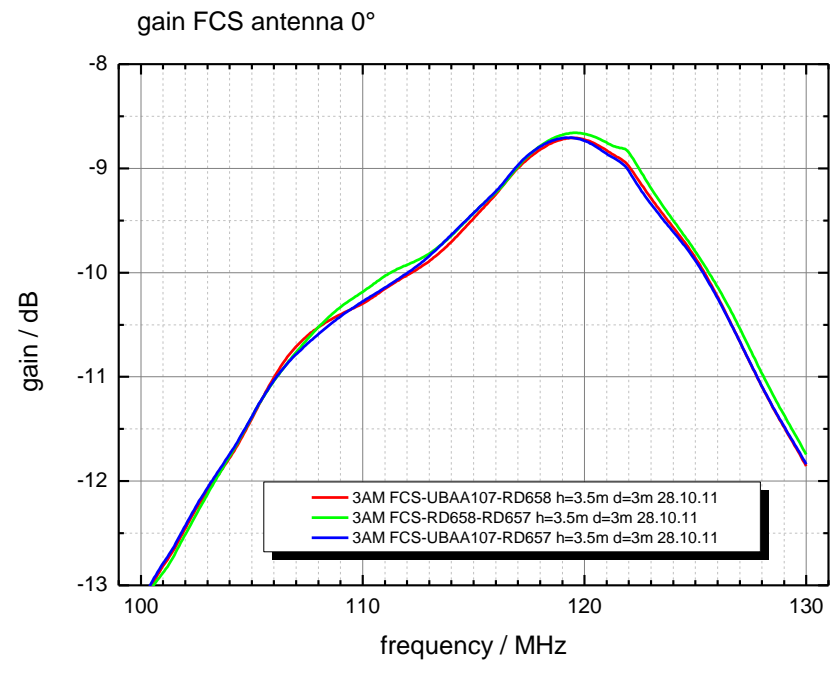

Fig. 4. Antenna gain for the AUT (FCS) obtained using reference dipoles (RD) and Schwarzbeck biconical antennas type UBAA in $3 \mathrm{~m}$ distance.

\subsection{Validation of the antenna calibration}

After this validation we used the 3-AM to determine the gain and subsequentially the antenna factor of the unknown reference antenna under test (AUT; cp. to FCS in Fig. 4) from two sets of three pairs of measurements. The first set included the two reference dipoles and the AUT, the second set included one reference dipole, a biconical antenna, and the AUT. Taking the antenna gain of one of the reference dipoles from (a) the initial validation (cp. Sect. 3.1), (b) from the first set of measurements, (c) from the second set of measurements, and (d) from theoretical calculations, we estimated the influence of our AUT on the measurements. The deviations found are within the specified uncertainty range (cp. Fig. 4). Now, we compared the results from several measurements of antenna gain for a biconical antenna, which is slightly larger than a reference dipole. We used the gain a) from the second set here, (b) from the initial validation (cp. Sect. 3.1), and (c) from the external calibration certificate. Again, the deviations found are within the specified uncertainty range (cp. Fig. 4).

Thus, we have experimentally proven, that the AUT does not change the measurement conditions in an unacceptable manner, and, furthermore, that the antenna gain obtained in this particular setup gives the best estimate for the gain of the AUT.

\section{Measurement uncertainty}

In order to estimate the overall uncertainty we had to derive the measurement equation first. In a second step we identified the contributions to the uncertainty and determined where they come into effect. We take also into account the steps to ensure traceability to the SI units. The uncertainty 
budget was derived according to JCGM (2008) using the GUM Workbench Pro tool (Metrodata, 2010). The contributions to uncertainty affect mainly the measurements of the forward transmission. In order to investigate the error propagation we revised the terms $a_{j} a_{k}$ in Eq. $(2 \mathrm{a}-\mathrm{c})$ to

$$
\begin{aligned}
& \left(a_{j} a_{k}\right)^{\prime}=a_{j} a_{k}+d_{\mathrm{SI}}+d_{\text {Cable }}+d_{\text {Misalign }} \\
& +d_{\text {Reflex }}+d_{\text {Multi }}+d_{\text {Mismatch }}+d_{\text {Repeat }}
\end{aligned}
$$

In Table 1 we specified all contributions to the overall uncertainty, in which $d_{\mathrm{SI}}$ is the uncertainty due to traceability to SI, $d_{\text {Cable }}$ is the influence of the cable movement on the measurement of the S-parameter, $d_{\text {Misalign }}$ is the misalignment of the antennas, $d_{\text {Reflex }}$ is the remaining reflectivity of the environment, $d_{\text {Multi }}$ are multiple reflections between the antennas, $d_{\text {Mismatch }}$ is the influence of varying impedance levels by cable connections, $d_{\text {Repeat }}$ takes into account the repeatability of the used $\mathrm{N}$ connector. For the final application, the anisotropy of the sensitivity diagram $\left(d_{\text {Aniso }}\right)$ has to be taken into account. This will affect the actual gain of the antenna in Eq. (4). Correlations are not taken into account.

The best estimate for the correction terms in Eq. (6) is $0 \mathrm{~dB}$, but we can associate the uncertainty to each contribution. Please note, that all three $a_{j} a_{k}$ need to be revised as the influences occur again in each pairing of the antennas. The drift of the VNA was negligible compared to the deviation we introduced for traceability to the SI units $\left(d_{\mathrm{SI}}\right)$. The reason is that we performed validation measurements before and after the measurement campaigns, which would reveal any drift by the VNA, provided that the artefacts like attenuators and mismatches remain stable. Cable movements during the measurement campaign, e.g. when the antennas are moved up to $3.5 \mathrm{~m}$ height, could result in long-term stress and phase shifts, which would also be revealed by measuring the artefacts at the ends of both cables. To obtain as much insight as possible into the measurement setup, these measurements should be taken on the ground and when the cable end including the artefact (mismatch) is moved up along the mast to the actual measurement position. Using some examples for measured data the expanded uncertainty (using $k=2$ ) for the antenna factor was calculated to $1 \mathrm{~dB} \mathrm{~m}^{-1}$.

\section{Application of the calibration factor}

The reference antenna was designed as a validation tool for absolute electrical field strength measurements on the localizer transmitter of instrument landing systems. In particular, measurements obtained during regular flight inspection are to be verified applying a method which is totally independent from FI aircrafts and their instrumentation.

Therefore, the final step was to compare the reference power density measurements employing the new antenna with those obtained with a computed (MLFMA) 3-D antenna pattern (Bredemeyer, 2007) as of the flight inspection (FI) aircraft.
Two measurement campaigns were carried out at Braunschweig (EDVE) and Bückeburg (ETHB) airports to cover the lower $(108.5 \mathrm{MHz})$ and the upper $(111.55 \mathrm{MHz})$ frequency ranges. The helicopter was deployed at various positions which are subsequently passed by in periodic flight inspection missions. Those power densities gained with the traceable reference antenna were then compared with the most recent flight inspection results, using the aircraft's VHF top dipole for comparison. Measurements at Braunschweig are shown in the above two diagrams. An ILS approach on centerline with the corresponding power density is depicted by Fig. 5. At ILS Point "A" 4 NM (nautic miles) before threshold a value $-77.5 \mathrm{dBW} \mathrm{m}^{-2}$ can be read. On the orbital flight with the aircraft at $7 \mathrm{NM}$ distance and $1800 \mathrm{ft}$ (feet) altitude a value of about $-100 \mathrm{dBW} \mathrm{m}^{-2}$ is given in Fig. 6 at $-10^{\circ}$ offset from the LOC antenna.

Since it is not a fixed assembly, the reference antenna may either rotate horizontally or swing laterally. The former is without influence due to the antenna's omnidirectional pattern. The latter is compensated by monitoring the maximum swings of the received level. This is included as an additional input to the overall measurement uncertainty budget (see section below).

The helicopter measurement values are depicted in the red curves (left Y-axis) in diagrams of Fig. 7. Within the marked areas (blue circles) the helicopter was kept relatively stable along a period of time (X-axis) and clear maximum power densities can be traced. On the right Y-axis the absolute 3$\mathrm{D}$ velocity according to the GPS receiver (green curve) is mapped. Depending on the air speed and the pilot's flight control the absolute speed (vertical and ground) may vary.

From the reference measurements a value of $79.5 \mathrm{dBW} \mathrm{m}^{-2}$ is obtained at ILS point A (see Fig. 7, left diagram), which is $2 \mathrm{~dB}$ below the aircraft result. From the helicopter measurements on approach 26 and orbit 7 NM we get a maximum of roughly $-99 \mathrm{dBW} \mathrm{m}^{2}$ in the highlighted area (cp. Fig. 7, right diagram), which is $1 \mathrm{~dB}$ above the aircraft measurements.

The results obtained at Bückeburg airport showed deviations in the same order of magnitude.

\section{Conclusions}

We presented a method for the traceable calibration of a newly designed horizontally polarized and omnidirectional antenna, which is used for the validation of electrical field strength measurements on instrument landing systems (ILS), in particular for the localizer transmitter. ILS' are subject to regular flight inspections (FI), which are performed using midsized aircrafts. In order to obtain absolute values of the field strength levels, aircrafts and their instrumentation need a thorough calibration, e.g. a 3-D-antenna pattern diagram has to be determined as a function of frequency. Of course, this is not an easy task as the calibration would be performed 


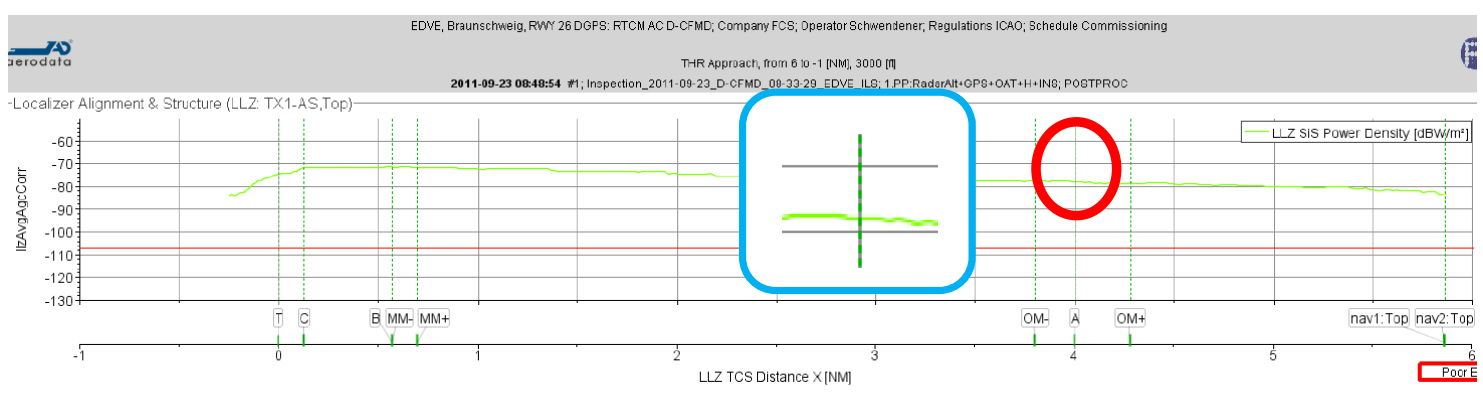

Fig. 5. Flight inspection aircraft: LOC Power Densities on Approach 26 at Braunschweig.

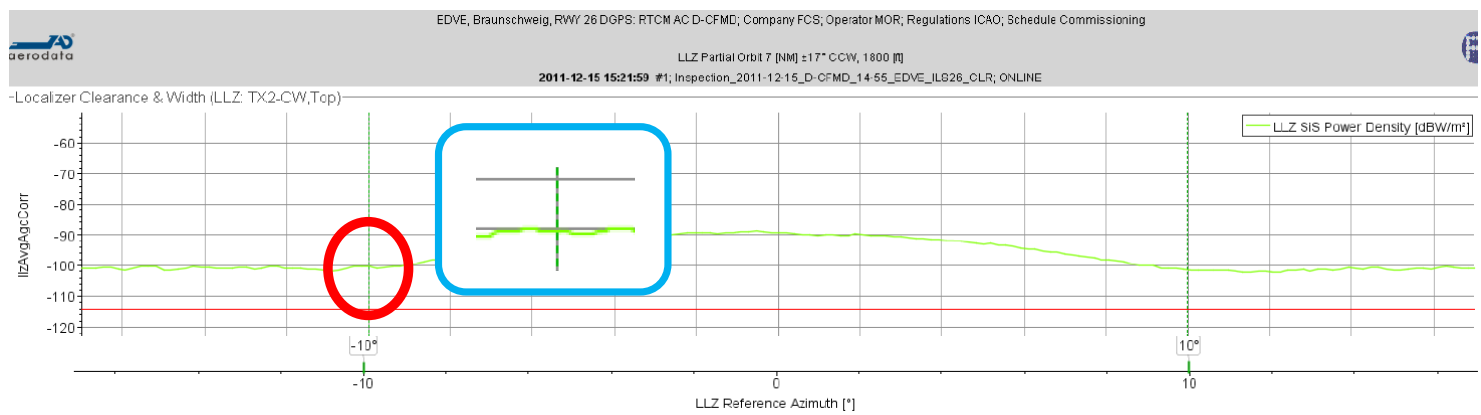

Fig. 6. LOC Power Densities on Orbital Flight 7NM at Braunschweig.

on the ground level, but is actually needed for the case that the aircraft is performing measurements in free-space and at many different bank angles (Bredemeyer, 2007).

To validate FI measurement results obtained with such flight inspection aircrafts, a new method was presented recently (Bredemeyer et al., 2012). This employs a newly designed reference antenna and a receiver system which are carried by a helicopter on its external load hook. Again, for absolute field strength measurements using this reference antenna, a suitable calibration for it as AUT (antenna under test) is required as well. Due to its more magnetic type of functionality, we had to verify that we could perform a typical 3-antenna-method without sacrificing the results. Another issue to be solved was the applicability of our anechoic chamber. Its design does not meet very stringent and specific requirements for antenna calibrations. In order to investigate these two issues we applied the 2-antenna-method and the 3-antenna-method for several pairs of antennas. These comprise a set of reference dipoles and broadband biconical antennas, for which we hold calibration certificates from several ISO/DIN/EN 17025 accredited calibration laboratories. We then compared theoretical and measured data sets of the dipoles and actual measurement results of the biconical antennas with results from calibration certificates applying the $\mathrm{E}_{n}$-criteria. All measurement results are based on scattering parameter measurements employing a vector network analyser.

\section{Results A}

Firstly, we were able to reproduce both the theoretical antenna factors of the reference dipoles and the antenna factors calculated from the antenna gain stated in the calibration certificates of the biconical antennas. Hence, we have experimentally verified that the setup in our anechoic chamber allows for this particular antenna calibration within the specified uncertainty range.

\section{Results B}

Secondly, we performed a 3-antenna-method comprising the new reference antenna, a reference dipole and a biconical antenna, whose calibration factor is known from external - but traceable - calibration. Comparing the actual antenna gains with the theoretical and the external results, we found good agreement within the specified range of uncertainty. Moreover, the antenna gains of the reference dipole and of the biconical antenna were in good agreement comparing the results from measurement campaign A and B (cp. results A and $\mathrm{B}$ in this section). Thereby, we experimentally verified the applicability of the 3-antenna-method on this type and size of the AUT within the specified range of uncertainty. The overall uncertainty was calculated to $1 \mathrm{~dB} \mathrm{~m}^{-1}$. 
Table 1. Uncertainty budget

\begin{tabular}{|c|c|c|c|c|c|c|}
\hline Quantity & Value & Standard Uncertainty & Distribution & Sensitivity Coefficient & Uncertainty Contribution & Index \\
\hline lambda & $2.7253859818 \mathrm{~m}$ & $14.3 \cdot 10^{-9} \mathrm{~m}$ & & & & \\
\hline$c$ & $299.792458 \cdot 10^{6} \mathrm{~m} \mathrm{~s}^{-1}$ & & & & & \\
\hline$f$ & $110.000000000 \cdot 10^{6} 1 \mathrm{~s}^{-1}$ & $0.5771 \mathrm{~s}^{-1}$ & rectangular & $39 \cdot 10^{-9}$ & $23 \cdot 10^{-9} \mathrm{~dB} \mathrm{~m}^{-1}$ & $0.0 \%$ \\
\hline PL & $5.2263 \cdot 10^{-3} \mathrm{~m}^{2} \mathrm{~s}^{2}$ & $20.1 \cdot 10^{-6} \mathrm{~m}^{2} \mathrm{~s}^{2}$ & & & & \\
\hline PI & 3.1415926535898 & & & & & \\
\hline$R$ & $3.00000 \mathrm{~m}$ & $5.77 \cdot 10^{-3} \mathrm{~m}$ & rectangular & -1.4 & $-8.4 \cdot 10^{-3} \mathrm{~dB} \mathrm{~m}^{-1}$ & $0.0 \%$ \\
\hline$a_{1} a_{2}$ & $199.5 \cdot 10^{-6}$ & $37.3 \cdot 10^{-6}$ & & & & \\
\hline$a_{1} a_{2} \log$ & $-37.0 \mathrm{~dB}$ & & & & & \\
\hline$d_{\mathrm{SI}}$ & $0.0 \mathrm{~dB}$ & $0.300 \mathrm{~dB}$ & normal & -0.50 & $-0.15 \mathrm{~dB} \mathrm{~m}^{-1}$ & $9.1 \%$ \\
\hline$d_{\text {Cable }}$ & $0.0 \mathrm{~dB}$ & $0.404 \mathrm{~dB}$ & rectangular & -0.50 & $-0.20 \mathrm{~dB} \mathrm{~m}^{-1}$ & $16.5 \%$ \\
\hline$d_{\text {Misalign }}$ & $0.0 \mathrm{~dB}$ & $0.289 \mathrm{~dB}$ & rectangular & -0.50 & $-0.14 \mathrm{~dB} \mathrm{~m}^{-1}$ & $8.4 \%$ \\
\hline$d_{\text {Reflex }}$ & $0.0 \mathrm{~dB}$ & $0.404 \mathrm{~dB}$ & rectangular & -0.50 & $-0.20 \mathrm{~dB} \mathrm{~m}^{-1}$ & $16.5 \%$ \\
\hline$d_{\text {Multi }}$ & $0.0 \mathrm{~dB}$ & $0.289 \mathrm{~dB}$ & rectangular & -0.50 & $-0.14 \mathrm{~dB} \mathrm{~m}^{-1}$ & $8.4 \%$ \\
\hline$d_{\text {Mismatch }}$ & $0.0 \mathrm{~dB}$ & $0.212 \mathrm{~dB}$ & U-distr. & -0.50 & $-0.11 \mathrm{~dB} \mathrm{~m}^{-1}$ & $4.5 \%$ \\
\hline$d_{\text {Repeat }}$ & $0.0 \mathrm{~dB}$ & $0.173 \mathrm{~dB}$ & rectangular & -0.50 & $-0.087 \mathrm{~dB} \mathrm{~m}^{-1}$ & $3.0 \%$ \\
\hline$a_{1} a_{3}$ & $398.1 \cdot 10^{-6}$ & $74.5 \cdot 10^{-6}$ & & & & \\
\hline$a_{1} a_{3} \log$ & $-34.0 \mathrm{~dB}$ & & & & & \\
\hline$a_{2} a_{3}$ & $1.000 \cdot 10^{-3}$ & $187 \cdot 10^{-6}$ & & & & \\
\hline$a_{2} a_{3} \log$ & $-30.0 \mathrm{~dB}$ & & & & & \\
\hline$G_{1}$ & $-9.091 \mathrm{dBi}$ & $0.498 \mathrm{dBi}$ & & & & \\
\hline$d_{\text {Aniso }}$ & $0.0 \mathrm{~dB}$ & $0.289 \mathrm{~dB}$ & rectangular & -1.0 & $-0.29 \mathrm{~dB} \mathrm{~m}^{-1}$ & $33.6 \%$ \\
\hline$Z_{0}$ & $376.99 \mathrm{~V} / \mathrm{A}$ & & & & & \\
\hline$Z_{L}$ & $50.0 \mathrm{~V} / \mathrm{A}$ & & & & & \\
\hline $\mathrm{AF}$ & $20.148 \mathrm{~dB} \mathrm{~m}^{-1}$ & $0.498 \mathrm{~dB} \mathrm{~m}^{-1}$ & & & & \\
\hline
\end{tabular}

EDVE LOC 26 Appr Point "A"

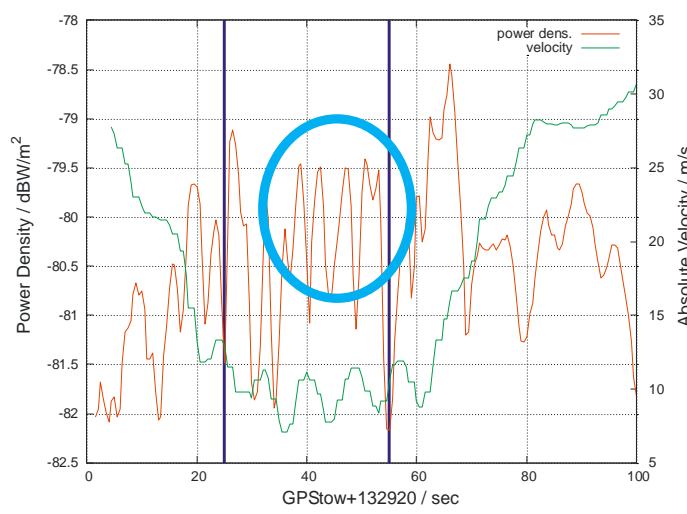

Fig. 7. Reference power densities on Approach 26 and Orbit 7NM.

\section{Practical application of the reference antenna}

In the first practical applications at different airports (Braunschweig and Bückeburg, Germany) we obtained measurement results of absolute electrical field strength levels from regular flight inspection employing the typical FI aircraft and from the measurement with the helicopter setup using the calibrated reference antenna. Some sample checks on different LOC frequencies were performed. This revealed a satisfactory agreement (max. deviation $2 \mathrm{~dB}$ ) between the power densities gained for the localizer transmitters of instrument

\section{EDVE LOC 26 Orbit 7NM -10 ${ }^{\circ}$}

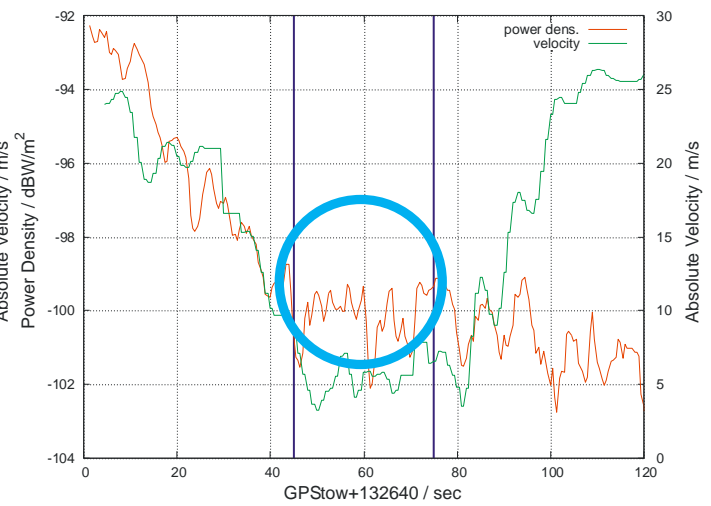

landing systems. Even despite the fact, that we have used two diametric airborne measurement setups on the same physical value, we found very satisfactory results. This ensures flight inspection and correspondent absolute electrical field strength measurements at VHF frequencies at a high level of confidence.

Acknowledgements. The authors thank Kai Baaske from PTB and Jörg Follop from FCS for their support during the measurement campaigns. 


\section{References}

Balanis, C. A., Antenna Theory, 2 Edn., New York: John Wiley \& Sons Inc., 1982.

Bredemeyer, J., Schrader, T., Kleine-Ostmann, T., and Garbe, H.: Quasi-stationary Signal-in-Space Measurements using Traceable Antennas, in: Proceedings of the 17th International Flight Inspection Symposium (IFIS) 2012, Braunschweig, Germany, 4-8 June, 2012.

Bredemeyer, J., Kleine-Ostmann, T., Schrader, T., Münter, K., and Ritter, J.: Airborne field strength monitoring, Adv. Radio Sci., 5, 49-55, doi:10.5194/ars-5-49-2007, 2007.

CISPR 16-1-5, Specification for radio disturbance and immunity measuring apparatus and methods - Part 1-5: Radio disturbance and immunity measuring apparatus - Antenna calibration test sites for $30 \mathrm{MHz}$ to $1000 \mathrm{MHz}, 2003$.

CISPR 16-1-6, Specification for radio disturbance and immunity measuring apparatus and methods - Part 1-6: Radio disturbance and immunity measuring apparatus - EMC-Antenna calibration, Draft standard, not published yet.
Hemming, L. H.: Electromagnetic Anechoic Chambers, IEEE Press, Canada, 2002.

ICAO Annex 10, Volume I, Radio Navigation Aids, Sixth Edition, July 2006.

ICAO DOC 8071, Volume I, Testing of Ground-based Radio Navigation Systems, Fourth Edition - 2000.

JCGM 100:2008. Evaluation of measurement data - Guide to the expression of uncertainty in measurement (GUM).

Metrodata GmbH, 79576 Weil am Rhein, Germany, GUM Workbench Pro tool, 2010.

Newell, A. C.: Error Analysis Techniques for Planar Near-Field Measurements, IEEE Trans. Antennas Propagation, 36, 254-268, June 1988, 1988.

Wöger, W., Remarks on the $\mathrm{E}_{n}$-Criterion Used in Measurement Comparisons, PTB-Mitteilungen, Braunschweig, 1, 24-27, 1999. 